\title{
Master switches of T-cell activation and differentiation
}

\author{
K.C. Beier, T. Kallinich and E. Hamelmann
}

ABSTRACT: T-cells play a central role in allergic airway diseases such as bronchial asthma. The imbalance between allergen-specific pro-inflammatory and pro-allergic T-cell responses on one hand and regulatory or suppressive T-cell responses on the other may best explain the development of unwanted immune responses against environmental allergens, which lead to immunoglobulin E production and airway inflammation. A key role in the fine tuning of any T-cell response is provided by the engagement of so-called co-stimulatory molecules that are required for the full activation of T-cells and the recognition of antigens via the antigen-specific T-cell receptor. Many of these co-stimulatory molecules have been identified only recently, leading to a fundamental change in the overall understanding of T-cell regulation.

Due to their pivotal impact on T-cell differentiation and control, co-stimulatory molecules are promising targets for therapeutic intervention in T-cell-regulated or -mediated immune disorders, including allergic diseases and asthma. In the present article, an attempt is made to summarise the current knowledge on the basic concept of co-stimulation, the presently known co-stimulatory molecules and their various functions on T-cell activation or suppression. The mini-series will be completed by two more articles describing the recent experimental studies and preliminary clinical findings regarding the role of co-stimulatory molecules in allergic disorders and bronchial asthma, and a discussion regarding the feasibility of co-stimulatory molecules as potential targets for the treatment of allergic airway disease.

Although it is too early for any clinical implication or utilisation at this moment, the authors are convinced that a better understanding of co-stimulation in the context of allergic asthma will finally provide novel and promising approaches for treatment and prevention.

KEYWORDS: Allergic immune response, allergic inflammation, bronchial asthma, co-stimulation, immune modulation, T-cell

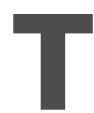
-cells play a central role in allergenmediated airway inflammation and disease. The prerequisite to any immune response against an allergen is its specific recognition, which, in the case of T-cell responses, is mediated by the antigen-specific T-cell receptor (TCR). However, the TCR-transmitted signal alone is insufficient to induce T-cell responses; it requires additional modulation by a constantly growing number of co-receptors with positive or negative regulatory function. Many of these co-stimulatory molecules have been identified only recently, leading to a fundamental change in the understanding of $\mathrm{T}$-cell regulation.

Considering their pivotal impact on T-cell regulation, co-stimulatory molecules are promising targets for therapeutic intervention in T-cellregulated or -mediated immune disorders, including allergic diseases. The aim of this mini-series of three articles is: 1) to give an overview of the current knowledge of the various co-stimulatory molecules; 2) to summarise the recent experimental studies and preliminary clinical findings regarding a role of co-stimulatory molecules in allergic disorders and bronchial asthma; and 3) to discuss the feasibility of costimulatory molecules as potential targets for the treatment of allergic airway disease.

\section{THE PRINCIPLES OF CO-STIMULATION}

Antigen-mediated immune responses are controlled and driven by the specific activation of T-cells. The "first signal" delivered by the TCR

\section{AFFILIATIONS}

Charité - Universitätsmedizin Berlin, Dept of Paediatric Pneumology and Immunology, Campus Virchow-

Klinikum, Berlin, Germany.

\section{CORRESPONDENCE}

E. Hamelmann

Dept of Paediatric Pneumology and Immunology

Charité - Universitätsmedizin Berlin Campus Virchow-Klinikum

Augustenburger Platz 1

13353 Berlin

Germany

Fax: 4930450566931

E-mail: eckard.hamelmann@charite.de

Received:

July 182006

Accepted after revision:

December 202006

STATEMENT OF INTEREST

None declared. 
mediates the specificity of a T-cell response via the recognition of specific epitopes of a given antigen presented in combination with the major histocompatibility complex on antigenpresenting cells (APCs). However, activation of T-cells by this receptor interaction alone fails to induce cytokine production and sustained proliferation, but rather results in T-cell apoptosis, or the induction of specific nonresponsiveness (anergy) to subsequent stimulation with the same antigen [1].

To become fully activated, T-cells require additional signals delivered by so-called co-stimulatory molecules (fig. 1a). These molecules are transmembrane proteins that induce an intracellular signalling cascade via their cytoplasmic tail that modifies the TCR-mediated signal. Co-stimulatory molecules cannot activate T-cells without concomitant TCR crosslinking. According to this definition, adhesion molecules such as intercellular adhesion molecule-1 are not co-stimulatory because they enhance T-cell activation merely by facilitating the contact between T-cell and APC. Furthermore, T-cell costimulation refers to a signal that is delivered to the T-cell exclusively. In this respect, the CD40/CD40L interaction is also not considered as co-stimulatory, although this is an important receptor/ligand pair in $\mathrm{T} / \mathrm{B}$ cooperation.

The dependence of T-cell activation on this "second signal" delivered by co-stimulators adds a second line of regulation to antigen-specific T-cell responses that reaches far beyond a mere "on-off" command. With a growing number of both stimulatory and inhibitory co-stimulatory molecules being identified, the classic concept of co-stimulation as a two-signal event has changed. T-cells simultaneously express an adjustable spectrum

a)

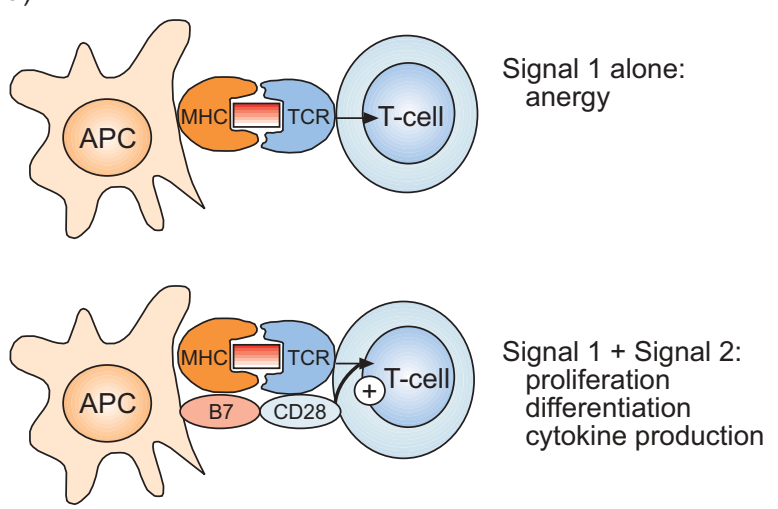

b)

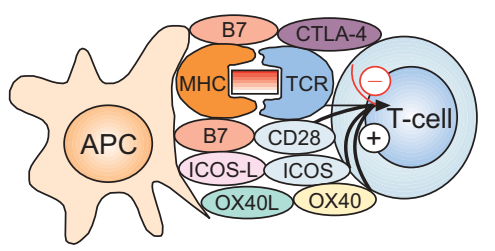

Integration of multiple positive and negative signals

FIGURE 1. a) Historic view and b) modern concept of co-stimulation. APC antigen-presenting cell; MHC: major histocompatibilty complex; TCR: T-cell receptor; CTLA: cytotoxic T-Iymphocyte antigen; ICOS: inducible co-stimulator; L: ligand. of co-stimulatory molecules. Today, T-cell co-stimulation is recognised as an integrating process of various positive and negative signals that determine the mode of $\mathrm{T}$-cell activation (as reviewed previously [2]; fig. 1b).

Co-stimulatory molecules can be categorised according to particular characteristics as follows.

\section{Expression pattern}

Only a few co-stimulatory molecules (CD28, CD27, herpes virus entry mediator (HVEM) and B- and T-lymphocyte attenuator (BTLA)) are expressed constitutively on both unstimulated and stimulated cells (constitutive expression). The majority of T-cell co-stimulators are induced upon T-cell activation (inducible expression; table 1). Some co-stimulatory molecules are downregulated upon repetitious stimulation. Therefore, T-cells, such as naïve, recently activated, or memory T-cells express a unique combination of co-stimulatory receptors depending on their history of activation and their state of differentiation.

\section{Expression density}

The number of copies of a co-stimulatory molecule expressed on the T-cell surface may have an impact on the modulating effect of T-cell effector function. For inducible co-stimulator (ICOS), for instance, it was demonstrated that a high-density expression was specifically associated with high production of interleukin (IL)-10, while an intermediate ICOS-expression density was correlated with the predominant production of T-helper cell (Th)2-type cytokines, IL-4, IL-5 and IL-13 [3].

\section{Positive versus negative signal}

Co-stimulatory molecules can modulate the TCR-mediated T-cell activation either negatively or positively (table 1). Most members of the constitutively expressed co-stimulators show positive amplifying effects, whereas the group of inducible costimulators contains both positive (ICOS, CD30, OX40, 4-1BB, signalling lymphocyte activation molecule (SLAM)) as well as negative (cytotoxic T-lymphocyte antigen (CTLA)-4, programmed death (PD)-1) regulators (table 1). Positive and negative regulatory co-stimulators may be expressed on the same cells either simultaneously or consecutively, which contributes to the complexity of this integral process.

\section{Co-stimulatory effect}

Co-stimulatory signals modify the effector phase of T-cell activation by inducing or suppressing the production of cytokines, by influencing cell survival or apoptosis, or by the upregulation of other cell surface molecules. Generally, the signalling can be regarded as one-way through the receptor on the T-cell side [2]. In recent years, it has been reported that for many co-stimulatory receptor-ligand pairs signals are also delivered through the ligand. However, the in vivo significance of this so-called back-signalling is poorly understood [4]. The different effects of the various co-stimulatory molecules on T-cell activation and function are discussed below in more detail.

\section{Availability of ligands}

The availability of the specific ligands for co-stimulatory molecules varies at different sites of T-cell activation. Some ligands, such as B7-1 and B7-2, which are the common ligands 


\begin{tabular}{|c|c|c|c|c|}
\hline Expression & Signal & Co-stimulatory molecule & Ligand & Superfamily \\
\hline \multirow{3}{*}{ Constitutive } & & $\mathrm{CD} 27$ & $\mathrm{CD} 70$ & TNF/TNFR \\
\hline & & HVEM & LIGHT, BTLA & TNF/TNFR, CD28/B7 \\
\hline & Negative & BTLA & HVEM & CD28/B7, TNF/TNFR \\
\hline \multirow{6}{*}{ Inducible } & & OX40 (CD134) & OX40L & TNF/TNFR \\
\hline & & 4-1BB (CD137) & 4-1BBL & TNF/TNFR \\
\hline & & CD30 & CD30L (CD153) & TNF/TNFR \\
\hline & & SLAM (CD150) & SLAM (CD150) & CD2 \\
\hline & Negative & CTLA-4 (CD152) & B7-1, B7-2 (CD80, CD86) & CD28/B7 \\
\hline & & PD-1 & PD-L1, PD-L2 & $\mathrm{CD} 28 / \mathrm{B} 7$ \\
\hline
\end{tabular}

TNF: tumour necrosis factor; R: receptor; HVEM: herpes virus entry mediator; LIGHT: homologous to lymphotoxin, inducible expression, competing for GpD of herpes virus, expressed on activated T-lymphocytes; BLTA: B- and T-lymphocyte attenuator; ICOS: inducible co-stimulator; L: ligand; SLAM: signalling lymphocyte activation molecule; CTLA: cytotoxic T-lymphocyte antigen; PD: programmed death; B7-H4 and - H3: B7 homologues 4 and 3. Modified from [2]

for CD28 and CTLA-4, are mostly restricted to specialised APCs such as dendritic cells, B-cells and macrophages. Other ligands can also be found on endothelial, epithelial and other nonlymphoid tissues in the periphery. This suggests a basic difference in T-cell co-stimulation in lymphoid organs versus in the periphery (as reviewed previously [2]).

\section{CO-STIMULATORY MOLECULES}

With the rapid increase in knowledge of the function of a growing number of specific co-stimulatory molecules, costimulation is now starting to be recognised as the master switch for T-cell activation and modulation of T-cell function. In the following section, the latest facts about the currently known co-stimulatory molecules will briefly be summarised, with a focus on expression pattern, ligand interaction and function, if available.

\section{The CD28/B7 superfamily}

A common characteristic of the CD28 homologues is a single extracellular immunoglobulin (Ig) variable-like domain followed by a short cytoplasmic tail. The genes for CD28, CTLA-4 and ICOS are clustered in close proximity on chromosome 2 q33 [5], suggesting that they evolved from gene duplication. Sequence analysis showed that all three have an unpaired cysteine that allows them to homodimerise on the T-cell surface. In contrast, the genes for PD-1 and BTLA are separately located (on 2q37 and 3q13, respectively). PD-1 was shown to exist as a monomer on the cell surface and BTLA is likely to be expressed as a monomer because it also lacks the unpaired cysteine residue (fig. 2) [6].

Members of the CD28/B7 superfamily have key roles in regulating $\mathrm{T}$-cell activation and tolerance. The constitutively expressed CD28 and the inducible molecule ICOS provide critical positive second signals that promote and sustain T-cell responses. CTLA-4, PD-1 and BTLA deliver negative second signals attenuating or terminating an ongoing $\mathrm{T}$-cell response. CD28 and CTLA-4 share the ligand pair B7-1 and B7-2, while
ICOS and PD-1 bind to their own distinct ligands. BTLA is the only member of the Ig superfamily that binds to a member of the tumour necrosis factor receptor (TNFR) superfamily, namely HVEM (fig. 2). Furthermore, the identification of two orphan B7 homologues, $\mathrm{B} 7-\mathrm{H} 3$ and $\mathrm{B} 7-\mathrm{H} 4$, indicates that there are more pathways in the CD28/B7 family that are as yet unidentified.

\section{CD28}

CD28 is the most extensively studied member of the group of co-stimulatory molecules.

\section{Expression}

CD28 is constitutively expressed on almost all resting human CD4+ T-cells and $50-80 \%$ of all CD8+ T-cells. Chronic stimulation leads to downregulation of CD28 expression, suggesting a negative feedback mechanism limiting an overwhelming immune response [7].

\section{Ligands}

CD28 has two ligands, B7-1 (CD80) and B7-2 (CD86). Both are constitutively expressed at low levels on a subpopulation of specialised APCs and are rapidly upregulated on almost all APCs upon activation [8]. Both B7-1 and B7-2 can be expressed on activated T-cells [9]. However, the significance of their expression on T-cells is not yet well understood.

\section{Function}

Co-stimulation via CD28 is the most important pathway for the initial activation of naïve T-cells. Ligation of CD3 and CD28 promotes the production of IL- 4 and IL- 5 and seems to be unique in its ability to induce very high levels of IL-2 production, which enhances proliferation in an autocrine manner. Moreover, it provides resistance to apoptosis and induces long-term expansion of T-cells [10-12].

Cytotoxic T-lymphocyte antigen-4

CTLA-4 (CD152) is a potent inhibitor of T-cell activation. 


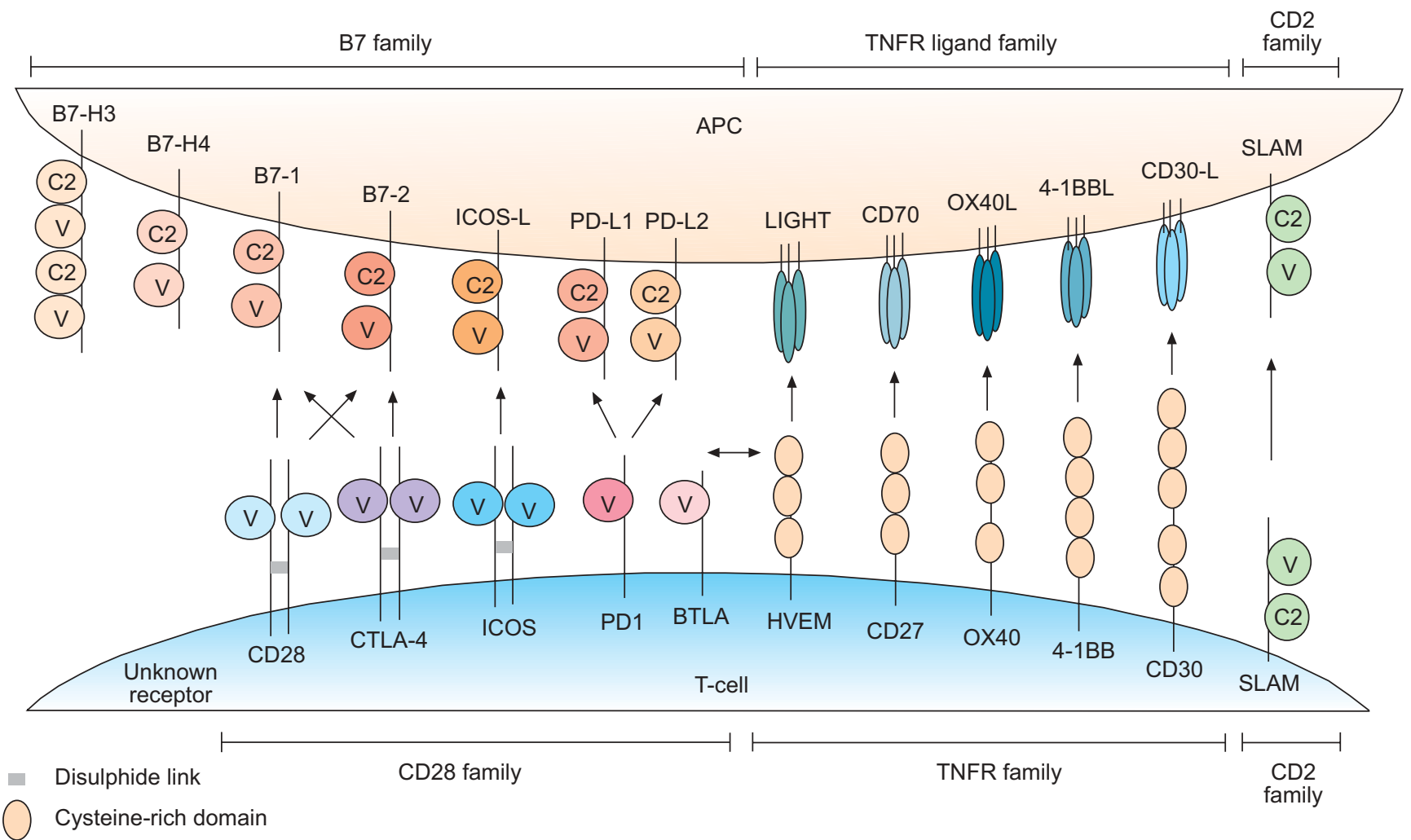

FIGURE 2. Currently known co-stimulatory molecules and their ligands. TNFR: tumour necrosis factor receptor; APC: antigen-presenting cell; B7-H3 and -H4: B7 homologues 3 and 4; ICOS: inducible co-stimulator; L: ligand; PD: programmed death; LIGHT: homologous to lymphotoxin, inducible expression, competing for GpD of herpes virus, expressed on activated T-lymphocytes; V: immunoglobulin-like variable domain; C2: constant type-2 immunoglobulin-like domain; SLAM: signalling lymphocyte activation molecule; CTLA: cytotoxic T-lymphocyte antigen; BTLA: B- and T-lymphocyte attenuator; HVEM: herpes virus entry mediator.

\section{Expression}

In contrast to CD28, CTLA-4 is only expressed after T-cell activation. It is usually located intracellularly and moves rapidly to the cell surface at the site of T-cell/APC interaction where it can inhibit the earliest events of T-cell activation $[13,14]$.

\section{Ligands}

Like CD28, CTLA-4 binds to the two ligands B7-1 and B7-2. CTLA-4 has higher affinity to B7-1 and B7-2 than CD28 and forms a lattice structure upon engagement with these ligands, which limits the ability of CD28 to interact with them [15]. CTLA-4 produces potent inhibitory effects on T-cell activation even at low expression levels.

\section{Function}

Besides ligand competition, CTLA-4 counteracts the positive signal of CD28 by the delivery of a negative signal that restricts autocrine IL-2 production and inhibits cell cycle progression. CTLA-4-deficient mice develop a fatal lymphoproliferative disease within the first few weeks of life, showing the importance of this molecule in the restriction of T-cellmediated immune responses [16].

Inducible co-stimulator

ICOS was the third member of the CD28 family to be discovered and is a positive regulator like CD28 [17, 18].

\section{Expression}

Unlike the constitutively expressed CD28, ICOS has to be induced by antigen stimulation, suggesting that ICOS provides signals to recently activated or memory rather than to naïve T-cells [17-19]. CD28 co-stimulation enhances ICOS expression, indicating that some of the functions ascribed to CD28 might be due in part to ICOS signalling [19-21]. However, ICOS expression is not entirely dependent upon CD28 signals, because CD28- CD8+ T-cells are able to express ICOS upon TCR stimulation [19]. ICOS is upregulated on both Th1 and Th2 cells during the initial phase of differentiation; expression levels remain high on Th2 cells but decrease on Th1 cells upon repetitive stimulation $[20,21]$.

\section{Ligand}

In contrast to CD28 and CTLA-4, ICOS binds to its own ligand, ICOS-L. ICOS-L is constitutively expressed at high density by B-cells, dendritic cells (DC) and macrophages, and also at low levels on T-cells. Moreover, ICOS-L is expressed on peripheral tissues (endothelium, brain, heart, liver and kidney) where it is upregulated by a variety of inflammatory signals [22-24].

\section{Function}

Like CD28, ICOS co-stimulation augments the production of effector cytokines such as IL-4, IL-5, interferon (IFN)- $\gamma$, and tumour necrosis factor (TNF)- $\alpha$. It is superior to CD28 in the 
enhancement of IL-10 production [17, 19]. Interestingly, ICOS co-stimulation promotes these effector-cell functions without enhancing IL-2 production. This lack of IL-2 production might provide a self-limiting mechanism to ICOS co-stimulation [25]. Besides its effects on T-cell responses, the ICOS/ICOS-L pathway seems to be most important for T-/B-cell cooperation regulating the production of $\operatorname{IgG}_{1}, \operatorname{IgG}_{2 a}$ and $\operatorname{IgE}$ in mice [20, $26,27]$, and the maintenance of the B-memory cell pool in humans [28]. This effect on B-cells seems to be due to the induction of cytokine secretion and the expression of other cell surface molecules on the T-cell by ICOS co-stimulation, rather than by direct signalling through ICOS-L.

\section{Programmed death-1}

PD-1 is the second negative regulatory member, besides CTLA-4, of the CD28/B7 family.

\section{Expression}

In contrast to other co-stimulatory molecules, PD-1 is not restricted to T-cells. It is also expressed on B-cells and myeloid cells following activation [29].

\section{Ligands}

PD-1 can bind two different ligands, PD-L1 (B7-H1) [30] and PD-L2 (B7-DC) [31, 32]. PD-L1 is constitutively expressed on freshly isolated T-cells, B-cells, macrophages and DCs, and was detected in the thymus as well as nonlymphoid tissues such as heart, kidney and liver. In contrast, PD-L2 is only expressed on activated macrophages and DCs [33]. These variations in tissue distribution and expression patterns suggest that the two PD-1 ligands play different roles at various stages of an immune response.

\section{Function}

Despite its name, PD-1 is associated with downmodulation of T-cell responses rather than apoptosis. It was shown that PD-1 leads to arrest in cell cycle phase G0-G1 [29]. CD28 costimulation or exogenous IL-2 can overcome the inhibitory effect of PD-1 on proliferation and cytokine production. Therefore, PD-1 effectively suppresses T-cell responses in situations in which IL-2 production is limited, as in the case of ICOS co-stimulation [34]. The importance of PD-1-mediated T-cell suppression for peripheral tolerance is indicated by the observation that PD-1-deficient mice develop auto-immune diseases [35, 36]. In contrast to CTLA-4-deficient mice, symptoms of auto-immunity develop later in life. This supports the hypothesis that CTLA- 4 is the primary negative co-stimulatory signal within the germinal centre reaction, whereas PD-1 controls the induction and maintenance of autoimmune processes in the periphery [37].

\section{The B- and T-lymphocyte attenuator}

BTLA is another inhibitory molecule in the CD28/B7 family with similarities to CTLA-4 and PD-1.

\section{Expression}

BTLA is expressed by nearly all lymphoid cells, including T-cells, B-cells and DCs [38, 39]. It is upregulated on T-cells upon activation [40].

\section{Ligand}

Recently, it has been shown that the receptor for BTLA is HVEM [41, 42]. Remarkably, this is the only CD28/B7 family member interacting with a member of the TNFR family.

\section{Function}

Co-stimulation by BTLA leads to downregulation of IL-2 production in vitro. Proliferation of BTLA-deficient T-cells is increased, and BTLA-deficient mice have increased specific antibody responses and enhanced sensitivity to experimental auto-immune encephalomyelitis. After polarisation of T-cells, BTLA is mainly expressed on Th1 cells, suggesting a role for BTLA in controlling Th1-biased immune responses [40].

\section{B7 homologue 3}

B7-H3, a recently identified member of the human B7 family, is an orphan ligand sharing $\sim 25 \%$ amino acid identity with other B7 family members.

\section{Expression}

B7-H3 is not detectable on peripheral blood mononuclear cells, although mRNA is found in various peripheral tissues and in several tumour cell lines. Expression of B7-H3 is induced on DCs and monocytes after activation by inflammatory cytokines or mitogens in vitro [43].

\section{Ligand}

Soluble B7-H3 protein binds a putative ligand on activated CD4+ and CD8+ T-cells that is distinct from CD28, CTLA-4, ICOS and PD-1 [43].

\section{Function}

The function of B7-H3 and its receptor is still unknown. Originally, the ligand for B7-H3 was described as co-stimulating the proliferation of both CD4+ and CD8+ T-cells, enhancing the induction of cytotoxic T-cells, and increasing IFN- $\gamma$ production by activated T-cells in vitro. However, analysis of B7-H3-deficient mice indicated an inhibitory effect on T-cell function [44].

\section{B7 homologue 4}

Also known as B7x and B7S1, B7-H4 is yet another (and the most recently described) member of the B7 family of costimulatory proteins.

\section{Expression}

B7-H4 has a very broad tissue distribution. It is expressed on epithelial cells and has now been discovered to be inducible on T-cells, B-cells, DCs, and monocytes upon in vitro stimulation [45].

\section{Ligand}

Studies of the B7-H4 fusion protein showed that the putative receptor of $\mathrm{B} 7-\mathrm{H} 4$ is upregulated on activated T-cells [46].

\section{Function}

Triggering of the B7-4 ligand on T-cells with soluble B7-H4 fusion protein has a profound inhibitory effect on growth, cytokine secretion and the development of cytotoxicity. Administration of B7-H4 Ig into mice impairs antigen-specific 
T-cell responses, whereas the blockade of endogenous B7-H4 by specific monoclonal antibody promotes T-cell responses [46].

\section{The TNFR superfamily}

Several members of the TNF/TNFR superfamily have costimulatory function and influence $\mathrm{T}$-cell activation and differentiation in a number of ways. A common feature of the members of this family of transmembrane glycoproteins is cysteine-rich repeats of $\sim 40$ amino acids in the extracellular amino terminal. Some sequence similarities in the cytoplasmic regions may also occur (fig. 2). Some members of this family possess a region of homology in their intracellular domains known as the death domain. This domain derives its name from the fact that it is involved in signalling processes that ultimately lead to programmed cell death by apoptosis. Molecules of this family are either induced or highly upregulated on the T-cell surface after recognition of antigen. Thus, they seem to regulate cell proliferation and/or prevent unnecessary cell death after initial induction of the immune response. This process of co-stimulation may, therefore, control the absolute number of effector T-cells and regulate the frequency of memory T-cells that subsequently develop during a given antigen-mediated response. The constitutively expressed molecules CD27 and HVEM, as well as the inducible OX40 (CD134), CD30 and 4-1BB (CD137) will be introduced as follows.

\section{OX40 (CD134)}

Expression

OX40 is primarily expressed on activated T-cells of the CD4 phenotype with a bias for Th2 cells [47]. Antigen-experienced T-cells re-express OX40 rapidly within $4 \mathrm{~h}$ upon re-stimulation [48].

\section{Ligand}

The ligand for OX40, OX40L, is expressed on activated B-cells, DCs and endothelial cells [49].

\section{Function}

OX40 ligation induces cytoplasmic expression of B-cell leukaemia/lymphoma 2 gene product $(\mathrm{Bcl})-\mathrm{xL}$ and $\mathrm{Bcl}-2$, thus delivering anti-apoptotic signals and playing a role in T-cell survival [50]. Several studies of OX40- and OX40L-deficient mice have been performed, showing that OX40/OX40L signalling plays a major role in T-cell function in antiviral, as well as allergen-mediated immune responses (as previously reviewed [51]). It was shown that OX40 co-stimulation is not only necessary to accumulate effector T-cells during primary immune responses but also influences subsequent formation of memory cells [48].

\section{CD30}

Expression

CD30 is expressed on activated T-cells with a strong bias for Th2 [47], and on B-cells, natural killer (NK) cells and eosinophils [52].

\section{Ligand}

CD30L is expressed in inflamed peripheral tissues and resting $\mathrm{B}$-cells, as well as on activated T-cells so that interaction among T-cells can occur via CD30/CD30L [52]. Like OX40L, CD30L is found in areas of T-cell-B-cell contact, where it appears to maintain the survival of primed and memory T-cells [52].

\section{Function}

CD30 is inducible by TCR signalling in a CD28-dependent manner or by activation via IL-4 [53]. In terms of its preferential expression on Th2 cells, CD30 is similar to OX40. Triggering of T-cells via CD30 results in the predominant generation of Th2 cytokines [54]. Like OX40, CD30 seems to be important for T-cell memory and the humoral immune response [55].

\section{4-1BB (CD137)}

Expression

$4-1 \mathrm{BB}$ is rapidly induced after activation of T-cells, B-cells, monocytes and nonlymphoid tissues [56].

\section{Ligand}

4-1BBL is expressed on B-cells, monocytes and DCs upon activation [57]. In vitro, 4-1BBL can activate both CD4 and CD8 T-cells. In vivo, however, agonistic anti-4-1BB antibodies show a preferential effect on CD8 T-cells [52].

\section{Function}

A major effect of $4-1 \mathrm{BB}$ appears to be directed towards sustaining T-cell survival. 4-1BB engagement prevents activation-induced cell death by induction of Bcl-xL and Bfl-1 (a Bcl-2 homologue first described in human foetal liver), two pro-survival members of the Bcl-2 family [52].

\section{CD27}

\section{Expression}

CD27 is expressed constitutively on T-cells, NK cells and B-cells [58]. In humans, CD27 expression increases transiently with activation and is subsequently downregulated on T-effector cells after several rounds of cell division, in a similar manner to CD28 [52].

\section{Ligand}

CD70, which is the ligand for CD27, is found on activated T-cells, B-cells and DCs. CD27 signalling in B-cells also plays a direct role in B-cell memory commitment and differentiation and contributes to germinal centre formation [59].

\section{Function}

CD27 engagement does not co-stimulate high levels of IL-2 production by T-cells, but rather induces TNF at comparable levels to that induced by CD28 engagement. CD27 promotes the development of cytotoxic T-lymphocyte effectors, and enhances T-cell survival [60] (as reviewed previously [59]). CD27 expression may divide the memory T-cell population into two groups: effector cells lacking CD27 display a high antigen recall response, whereas $\mathrm{CD} 27+$ memory $\mathrm{T}$-cells require additional co-stimulation for $\mathrm{T}$-cell receptor triggering [61]. Mice overexpressing the natural CD27-ligand, CD70, showed a progressive conversion of naïve T-cells into effectormemory cells, which finally led to the loss of naïve T-cells in lymph nodes and the spleen [62].

\section{HVEM}

Expression

HVEM is expressed on T-cells constitutively and may be expressed upon stimulation by DCs, NK cells and on memory and naïve B-cells from peripheral blood or the tonsils, but not on germinal centre B-cells. 


\section{Ligand}

HVEM transmits a positive co-stimulatory signal by interaction with LIGHT (homologous to lymphotoxin, inducible expression, competing for $\mathrm{GpD}$ of herpes virus, expressed on activated T-lymphocytes), a molecule of the TNFR ligand family. Interestingly, it may also interact with the inhibitory molecule BTLA, which belongs to the CD28/B7 family [41, 42] and can bind secreted lymphotoxin [63].

\section{Function}

The HVEM-LIGHT interaction is thought to deliver a positive co-stimulatory signal for the cell expressing HVEM; this is based on the signalling profile of HVEM and the ability of soluble LIGHT to augment T-cell proliferation and cytokine secretion [64]. Due to spatial binding characteristics of BTLA versus LIGHT, it might be possible that only the binding of LIGHT induces a co-stimulatory signal, while BTLA binds competitively, reducing positive signals through HVEM and inducing inhibitory signals through BTLA [64]. Stimulation of B-cells with LIGHT-expressing T-cells increased B-cell proliferation induced by the CD40/CD40L interaction and IgG and $\operatorname{IgM}$ (but not $\operatorname{Ig} \mathrm{A}$ ) secretion [65].

\section{Other co-stimulatory molecules}

The signalling lymphocyte activation molecule SLAM (CD150) is a self-ligand cell surface glycoprotein and belongs to a new family of co-stimulatory molecules [66].

\section{Expression}

SLAM is expressed on activated T-cells, B-cells, macrophages and DCs [66]. Cell-surface expression is upregulated with rapid kinetics in activated T-cells and lipopolysaccharide/IFN- $\gamma$ activated macrophages.

\section{Ligand}

Interestingly, SLAM interacts with the identical molecules on partner cells in a homotypic way.

\section{Function}

It was initially shown that SLAM co-stimulation promotes proliferation and production of Th1 cytokines in vitro $[67,68]$. In vivo experiments with cell lines, however, led to conflicting results and require further investigation in knock-out mice [66].

\section{CONCLUDING REMARKS}

The modern concept of co-stimulation has shed new light on the mechanisms involved in the regulation of $\mathrm{T}$-cell activation and differentiation. The identification of several new costimulatory molecules and their receptors supports the idea of fine-tuning T-cell functions via a multitude of simultaneously or consecutively expressed T-cell molecules. Not only does the joint action of various co-stimulatory receptors on T-cells add to the complexity of this mechanism but also to the availability of ligands on different cell types and in different organs. The identification of the orphan ligands B7 homologue 3 and B7 homologue 4 shows that there may be more costimulatory molecules to be identified. Table 2 categorises the currently known co-stimulatory molecules that were introduced in the article according to functional aspects. With a growing insight into co-stimulatory mechanisms, a vast array of potential targets for the modulation and redirection of T-cell responses becomes available. Accordingly, many experimental studies using animal models have explored the efficacy of targeting co-stimulatory molecules for the inhibition, or even prevention of, the development of the different diseases. Among these, allergen-induced sensitisation and airway disease are of the most interest, because they are clearly mediated by misled T-cell responses against common environmental antigens. Most of the aforementioned molecules have been studied in murine models of allergic airway inflammation. The following article of this mini-series will give, therefore, an overview on these studies in murine models as well as discuss preliminary data in the human system. Finally, the third article will discuss the utilisation and feasibility of costimulatory molecules for novel treatment strategies of allergic airway disease.

\section{TABLE 2 Functional classification of co-stimulatory molecules}

\begin{tabular}{lcc} 
Functional aspect & Characterisation & Co-stimulatory molecule \\
\hline Expression pattern & $\begin{array}{c}\text { Constitutively } \\
\text { Inducible }\end{array}$ & CD28, CD27, HVEM, BTLA \\
T-cell modulation & Positive/enhancement & B \\
& Negative/inhibition & ICOS, CTLA-4, PD-1, OX40, 4-1BB, CD30, SLAM, putative receptors for \\
T-cell differentiation & Th1 & CD28, ICOS, OX40, CD27, 4-1BB, CD30, HVEM, SLAM \\
& Th2 & CTLA-4, PD-1, BTLA, putative receptor for B7-H4 \\
T-cell function & Treg & $4-1 \mathrm{BB}$, SLAM, BTLA \\
Location of action & T effector/helper & ICOS, OX40, CD30 \\
& T memory & ICOS, CTLA-4, PD-1 \\
\hline
\end{tabular}

HVEM: herpes virus entry mediator; BTLA: B- and T-lymphocyte attenuator; ICOS: inducible co-stimulator; CTLA: cytotoxic T-lymphcyte antigen; PD: programmed death; SLAM: signalling lymphocyte activation molecule; $\mathrm{B} 7-\mathrm{H} 3$ and $-\mathrm{H} 4$ : B7 homologues 3 and 4; Th: T-helper cell; Treg: regulatory T-cell. 


\section{REFERENCES}

1 Mueller DL, Jenkins MK, Schwartz RH. Clonal expansion versus functional clonal inactivation: a costimulatory signalling pathway determines the outcome of $\mathrm{T}$ cell antigen receptor occupancy. Annu Rev Immunol 1989; 7: 445-480.

2 Kroczek RA, Mages HW, Hutloff A. Emerging paradigms of T-cell co-stimulation. Curr Opin Immunol 2004; 16: 321-327.

3 Lohning M, Hutloff A, Kallinich T, et al. Expression of ICOS in vivo defines CD4+ effector $\mathrm{T}$ cells with high inflammatory potential and a strong bias for secretion of interleukin 10. J Exp Med 2003; 197: 181-193.

4 Logue EC, Sha WC. CD28-B7 bidirectional signaling: a two-way street to activation. Nat Immunol 2004; 5: 1103-1105.

5 Sharpe AH, Freeman GJ. The B7-CD28 superfamily. Nat Rev Immunol 2002; 2: 116-126.

6 Zhang X, Schwartz JC, Guo X, et al. Structural and functional analysis of the costimulatory receptor programmed death-1. Immunity 2004; 20: 337-347.

7 Posnett DN, Edinger JW, Manavalan JS, Irwin C, Marodon G. Differentiation of human CD8 T cells: implications for in vivo persistence of CD8+. Int Immunol 1999; 11: 229-241.

8 Hathcock KS, Laszlo G, Pucillo C, Linsley P, Hodes RJ. Comparative analysis of B7-1 and B7-2 costimulatory ligands: expression and function. J Exp Med 1994; 180: 631-640.

9 Taylor PA, Lees CJ, Fournier S, Allison JP, Sharpe AH, Blazar BR. B7 expression on $\mathrm{T}$ cells down-regulates immune responses through CTLA-4 ligation via T-T interactions [corrections]. J Immunol 2004; 172: 34-39.

10 Harding FA, McArthur JG, Gross JA, Raulet DH, Allison JP. CD28-mediated signalling co-stimulates murine $\mathrm{T}$ cells and prevents induction of anergy in T-cell clones. Nature 1992; 356: 607-609.

11 Rulifson IC, Sperling AI, Fields PE, Fitch FW, Bluestone JA. CD28 costimulation promotes the production of Th2 cytokines. J Immunol 1997; 158: 658-665.

12 Jenkins MK, Taylor PS, Norton SD, Urdahl KB. CD28 delivers a costimulatory signal involved in antigen-specific IL-2 production by human T cells. J Immunol 1991; 147: 2461-2466.

13 Brunet JF, Denizot F, Luciani MF, et al. A new member of the immunoglobulin superfamily - CTLA-4. Nature 1987; 328: $267-270$.

14 Walunas TL, Lenschow DJ, Bakker CY, et al. CTLA-4 can function as a negative regulator of $\mathrm{T}$ cell activation. Immunity 1994; 1: 405-413.

15 Schwartz JC, Zhang X, Nathenson SG, Almo SC. Structural mechanisms of costimulation. Nat Immunol 2002; 3: 427-434.

16 Tivol EA, Borriello F, Schweitzer AN, Lynch WP, Bluestone JA, Sharpe AH. Loss of CTLA-4 leads to massive lymphoproliferation and fatal multiorgan tissue destruction, revealing a critical negative regulatory role of CTLA-4. Immunity 1995; 3: 541-547.

17 Hutloff A, Dittrich AM, Beier KC, et al. ICOS is an inducible T-cell co-stimulator structurally and functionally related to CD28. Nature 1999; 397: 263-266.
18 Yoshinaga SK, Whoriskey JS, Khare SD, et al. T-cell costimulation through B7RP-1 and ICOS. Nature 1999; 402: 827-832.

19 Beier KC, Hutloff A, Dittrich AM, et al. Induction, binding specificity and function of human ICOS. Eur J Immunol 2000; 30: 3707-3717.

20 McAdam AJ, Chang TT, Lumelsky AE, et al. Mouse inducible costimulatory molecule (ICOS) expression is enhanced by CD28 costimulation and regulates differentiation of CD4+ T cells. J Immunol 2000; 165: 5035-5040.

21 Coyle AJ, Lehar S, Lloyd C, et al. The CD28-related molecule ICOS is required for effective T cell-dependent immune responses. Immunity 2000; 13: 95-105.

22 Mages HW, Hutloff A, Heuck C, et al. Molecular cloning and characterization of murine ICOS and identification of B7h as ICOS ligand. Eur J Immunol 2000; 30: 1040-1047.

23 Ling V, Wu PW, Finnerty HF, et al. Cutting edge: identification of GL50, a novel B7-like protein that functionally binds to ICOS receptor. J Immunol 2000; 164: 1653-1657.

24 Khayyamian S, Hutloff A, Buchner K, et al. ICOS-ligand, expressed on human endothelial cells, costimulates Th1 and Th2 cytokine secretion by memory CD4+ T cells. Proc Natl Acad Sci USA 2002; 99: 6198-6203.

25 Riley JL, Blair PJ, Musser JT, et al. ICOS costimulation requires IL-2 and can be prevented by CTLA-4 engagement. J Immunol 2001; 166: 4943-4948.

26 Dong C, Juedes AE, Temann UA, et al. ICOS co-stimulatory receptor is essential for T-cell activation and function. Nature 2001; 409: 97-101.

27 Tafuri A, Shahinian A, Bladt F, et al. ICOS is essential for effective T-helper-cell responses. Nature 2001; 409: 105-109.

28 Grimbacher B, Hutloff A, Schlesier M, et al. Homozygous loss of ICOS is associated with adult-onset common variable immunodeficiency. Nat Immunol 2003; 4: 261-268.

29 Agata $Y$, Kawasaki A, Nishimura H, et al. Expression of the PD-1 antigen on the surface of stimulated mouse $\mathrm{T}$ and $\mathrm{B}$ lymphocytes. Int Immunol 1996; 8: 765-772.

30 Dong H, Zhu G, Tamada K, Chen L. B7-H1, a third member of the B7 family, co-stimulates T-cell proliferation and interleukin-10 secretion. Nat Med 1999; 5: 1365-1369.

31 Latchman Y, Wood CR, Chernova T, et al. PD-L2 is a second ligand for PD-1 and inhibits $\mathrm{T}$ cell activation. Nat Immunol 2001; 2: 261-268.

32 Tseng SY, Otsuji M, Gorski K, et al. B7-DC, a new dendritic cell molecule with potent costimulatory properties for $\mathrm{T}$ cells. J Exp Med 2001; 193: 839-846.

33 Yamazaki $\mathrm{T}$, Akiba $\mathrm{H}$, Iwai $\mathrm{H}$, et al. Expression of programmed death 1 ligands by murine T cells and APC. J Immunol 2002; 169: 5538-5545.

34 Bennett F, Luxenberg D, Ling V, et al. Program death-1 engagement upon TCR activation has distinct effects on costimulation and cytokine-driven proliferation: attenuation of ICOS, IL-4, and IL-21, but not CD28, IL-7, and IL-15 responses. J Immunol 2003; 170: 711-718.

35 Nishimura $H$, Nose $M$, Hiai $H$, Minato $N$, Honjo $T$. Development of lupus-like autoimmune diseases by disruption of the PD-1 gene encoding an ITIM motifcarrying immunoreceptor. Immunity 1999; 11: 141-151.

36 Nishimura H, Minato N, Nakano T, Honjo T. Immunological studies on PD-1 deficient mice: implication of PD-1 as 
a negative regulator for B cell responses. Int Immunol 1998; 10: 1563-1572.

37 Carreno BM, Collins M. The B7 family of ligands and its receptors: new pathways for costimulation and inhibition of immune responses. Annu Rev Immunol 2002; 20: 29-53.

38 Han P, Goularte OD, Rufner K, Wilkinson B, Kaye J. An inhibitory Ig superfamily protein expressed by lymphocytes and APCs is also an early marker of thymocyte positive selection. J Immunol 2004; 172: 5931-5939.

39 Hurchla MA, Sedy JR, Gavrieli M, Drake CG, Murphy TL, Murphy KM. B and T lymphocyte attenuator exhibits structural and expression polymorphisms and is highly induced in anergic CD4+ T cells. J Immunol 2005; 174: 3377-3385.

40 Watanabe N, Gavrieli M, Sedy JR, et al. BTLA is a lymphocyte inhibitory receptor with similarities to CTLA-4 and PD-1. Nat Immunol 2003; 4: 670-679.

41 Sedy JR, Gavrieli M, Potter KG, et al. B and T lymphocyte attenuator regulates $\mathrm{T}$ cell activation through interaction with herpesvirus entry mediator. Nat Immunol 2005; 6: 90-98.

42 Gonzalez LC, Loyet KM, Calemine-Fenaux J, et al. A coreceptor interaction between the CD28 and TNF receptor family members $\mathrm{B}$ and $\mathrm{T}$ lymphocyte attenuator and herpesvirus entry mediator. Proc Natl Acad Sci USA 2005; 102: 1116-1121.

43 Chapoval AI, Ni J, Lau JS, et al. B7-H3: a costimulatory molecule for T cell activation and IFN-gamma production. Nat Immunol 2001; 2: 269-274.

44 Suh WK, Gajewska BU, Okada H, et al. The B7 family member B7-H3 preferentially down-regulates $\mathrm{T}$ helper type 1-mediated immune responses. Nat Immunol 2003; 4: 899-906.

45 Tringler B, Zhuo S, Pilkington G, et al. B7-H4 is highly expressed in ductal and lobular breast cancer. Clin Cancer Res 2005; 11: 1842-1848.

46 Sica GL, Choi IH, Zhu G, et al. B7-H4, a molecule of the B7 family, negatively regulates $\mathrm{T}$ cell immunity. Immunity 2003; 18: 849-861.

47 Kim MY, Gaspal FM, Wiggett $\mathrm{HE}$, et al. $\mathrm{CD}^{+} \mathrm{CD}^{-}$ accessory cells costimulate primed CD4 T cells through OX40 and CD30 at sites where T cells collaborate with B cells. Immunity 2003; 18: 643-654.

48 Gramaglia I, Weinberg AD, Lemon M, Croft M. Ox-40 ligand: a potent costimulatory molecule for sustaining primary CD4 T cell responses. J Immunol 1998; 161: 6510-6517.

49 Stuber E, Neurath M, Calderhead D, Fell HP, Strober W. Cross-linking of OX40 ligand, a member of the TNF/NGF cytokine family, induces proliferation and differentiation in murine splenic B cells. Immunity 1995; 2: 507-521.

50 Rogers PR, Song J, Gramaglia I, Killeen N, Croft M. OX40 promotes $\mathrm{Bcl}-\mathrm{xL}$ and $\mathrm{Bcl}-2$ expression and is essential for long-term survival of CD4 T cells. Immunity 2001; 15: 445-455.

51 Croft M. Costimulation of $\mathrm{T}$ cells by OX40, 4-1BB, and CD27. Cytokine Growth Factor Rev 2003; 14: 265-273.

52 Watts TH. TNF/TNFR family members in costimulation of T cell responses. Annu Rev Immunol 2005; 23: 23-68.
53 Gilfillan MC, Noel PJ, Podack ER, Reiner SL, Thompson CB. Expression of the costimulatory receptor CD30 is regulated by both CD28 and cytokines. J Immunol 1998; 160: 2180-2187.

54 Tarkowski M. Expression and a role of CD30 in regulation of T-cell activity. Curr Opin Hematol 2003; 10: 267-271.

55 Gaspal FM, Kim MY, McConnell FM, Raykundalia C, Bekiaris V, Lane PJ. Mice deficient in OX40 and CD30 signals lack memory antibody responses because of deficient CD4 T cell memory. J Immunol 2005; 174: 3891-3896.

56 Schwarz H, Valbracht J, Tuckwell J, von Kempis J, Lotz M. ILA, the human 4-1BB homologue, is inducible in lymphoid and other cell lineages. Blood 1995; 85: 1043-1052.

57 Futagawa T, Akiba H, Kodama T, et al. Expression and function of $4-1 \mathrm{BB}$ and $4-1 \mathrm{BB}$ ligand on murine dendritic cells. Int Immunol 2002; 14: 275-286.

58 de Jong R, Loenen WA, Brouwer M, et al. Regulation of expression of CD27, a T cell-specific member of a novel family of membrane receptors. J Immunol 1991; 146: 2488-2494.

59 Borst J, Hendriks J, Xiao Y. CD27 and CD70 in T cell and B cell activation. Curr Opin Immunol 2005; 17: 275-281.

60 Hintzen RQ, Lens SM, Lammers K, Kuiper H, Beckmann $\mathrm{MP}$, van Lier RA. Engagement of CD27 with its ligand CD70 provides a second signal for T cell activation. J Immunol 1995; 154: 2612-2623.

61 Taraban VY, Rowley TF, O'Brien L, et al. Expression and costimulatory effects of the TNF receptor superfamily members CD134 (OX40) and CD137 (4-1BB), and their role in the generation of anti-tumor immune responses. Eur J Immunol 2002; 32: 3617-3627.

62 Tesselaar K, Arens R, van Schijndel GM, et al. Lethal T cell immunodeficiency induced by chronic costimulation via CD27-CD70 interactions. Nat Immunol 2003; 4: 49-54.

63 Mauri DN, Ebner R, Montgomery RI, et al. LIGHT, a new member of the TNF superfamily, and lymphotoxin $\alpha$ are ligands for herpesvirus entry mediator. Immunity 1998; 8: 21-30.

64 Croft M. The evolving crosstalk between co-stimulatory and co-inhibitory receptors: HVEM-BTLA. Trends Immunol 2005; 26: 292-294.

65 Duhen T, Pasero C, Mallet F, Barbarat B, Olive D, Costello RT. LIGHT costimulates CD40 triggering and induces immunoglobulin secretion; a novel key partner in $\mathrm{T}$ cell-dependent B cell terminal differentiation. Eur J Immunol 2004; 34: 3534-3541.

66 Veillette A, Latour S. The SLAM family of immune-cell receptors. Curr Opin Immunol 2003; 15: 277-285.

67 Castro AG, Hauser TM, Cocks BG, et al. Molecular and functional characterization of mouse signaling lymphocytic activation molecule (SLAM): differential expression and responsiveness in Th1 and Th2 cells. J Immunol 1999; 163: 5860-5870.

68 Howie D, Simarro M, Sayos J, Guirado M, Sancho J, Terhorst C. Molecular dissection of the signaling and costimulatory functions of CD150 (SLAM): CD150/SAP binding and CD150-mediated costimulation. Blood 2002; 99: 957-965. 